Article

\title{
Connection Problem for Sums of Finite Products of Chebyshev Polynomials of the Third and Fourth Kinds
}

\author{
Dmitry Victorovich Dolgy ${ }^{1}$, Dae San Kim ${ }^{2}$, Taekyun Kim ${ }^{3,4}$ and Jongkyum Kwon ${ }^{5, *}$ \\ 1 Institute of National Sciences, Far Eastern Federal University, 690950 Vladivostok, Russia; d_dol@mail.ru \\ 2 Department of Mathematics, Sogang University, Seoul 04107, Korea; dskim@sogang.ac.kr \\ 3 Department of Mathematics, College of Science, Tianjin Polytechnic University, Tianjin 300160, China; \\ tkkim@kw.ac.kr \\ 4 Department of Mathematics, Kwangwoon University, Seoul 01897, Korea \\ 5 Department of Mathematics Education and ERI, Gyeongsang National University, \\ Jinju 52828, Gyeongsangnamdo, Korea \\ * Correspondence: mathkjk26@gnu.ac.kr; Tel.: +82-(0)10-8978-73576
}

Received: 8 October 2018; Accepted: 5 November 2018; Published: 9 November 2018

check for updates

\begin{abstract}
This paper treats the connection problem of expressing sums of finite products of Chebyshev polynomials of the third and fourth kinds in terms of five classical orthogonal polynomials. In fact, by carrying out explicit computations each of them are expressed as linear combinations of Hermite, generalized Laguerre, Legendre, Gegenbauer, and Jacobi polynomials which involve some terminating hypergeometric functions ${ }_{2} F_{0,2} F_{1}$, and ${ }_{3} F_{2}$.
\end{abstract}

Keywords: sums of finite products of Chebyshev polynomials of the third and fourth kinds; Hermite; generalized Laguerre; Legendre; Gegenbauer; Jacobi

MSC: 11B83; 33C05; 33C20; 33C45

\section{Introduction and Preliminaries}

In this section, we will recall some basic facts about relevant orthogonal polynomials that will be needed throughout this paper. For this, we will first fix some notations. For any nonnegative integer $n$, the falling factorial polynomials $(x)_{n}$ and the rising factorial polynomials $\langle x\rangle_{n}$ are respectively given by

$$
\begin{gathered}
(x)_{n}=x(x-1) \cdots(x-n+1),(n \geq 1),(x)_{0}=1, \\
<x>_{n}=x(x+1) \cdots(x+n-1),(n \geq 1),<x>_{0}=1 .
\end{gathered}
$$

The two factorial polynomials are evidently related by

$$
\begin{gathered}
(-1)^{n}(x)_{n}=<-x>_{n},(-1)^{n}<x>_{n}=(-x)_{n} . \\
\frac{(2 n-2 s) !}{(n-s) !}=\frac{2^{2 n-2 s}(-1)^{s}<\frac{1}{2}>_{n}}{<\frac{1}{2}-n>_{s}},(n \geq s \geq 0) . \\
B(x, y)=\int_{0}^{1} t^{x-1}(1-t)^{y-1} d t=\frac{\Gamma(x) \Gamma(y)}{\Gamma(x+y)},(\text { Re } x, \text { Re } y>0) . \\
\Gamma\left(n+\frac{1}{2}\right)=\frac{(2 n) ! \sqrt{\pi}}{2^{2 n} n !},(n \geq 0) .
\end{gathered}
$$




$$
\frac{\Gamma(x+1)}{\Gamma(x+1-n)}=(x)_{n}, \frac{\Gamma(x+n)}{\Gamma(x)}=<x>_{n},(n \geq 0),
$$

where $\Gamma(x)$ and $B(x, y)$ are the gamma and beta functions respectively.

The hypergeometric function is defined by

$$
\begin{aligned}
& p F_{q}\left(a_{1}, \cdots, a_{p} ; b_{1}, \cdots, b_{q} ; x\right) \\
& =\sum_{n=0}^{\infty} \frac{<a_{1}>_{n} \cdots<a_{p}>_{n}}{<b_{1}>_{n} \cdots<b_{q}>_{n}} \frac{x^{n}}{n !} .
\end{aligned}
$$

We are now ready to state some basic facts about Chebyshev polynomials of the third kind $V_{n}(x)$, those of the fourth kind $W_{n}(x)$, Hermite polynomials $H_{n}(x)$, generalized (extended) Laguerre polynomials $L_{n}^{\alpha}(x)$, Legendre polynomials $P_{n}(x)$, Gegenbauer polynomials $C_{n}^{(\lambda)}(x)$, and Jacobi polynomials $P_{n}^{(\alpha, \beta)}$. Chebyshev polynomials are diversely used in approximation theory and numerical analysis, Hermite polynomials appear as the eigenfunctions of the harmonic oscillator in quantum mechanics, Laguerre polynomials have important applications to the solution of Schrödinger's equation for the hydrogen atom, Legendre polynomials can be used to write the Coulomb potential as a series, Gegenbauer polynomials play an important role in the constructive theory of spherical functions and Jacobi polynomials occur in the solution to the equations of motion of the symmetric top. All the necessary facts on those special polynomials can be found in [1-9]. For the full accounts of this fascinating area of orthogonal polynomials, the reader may refer to [10-13].

The above special polynomials are given in terms of generating functions by

$$
\begin{gathered}
F(t, x)=\frac{1-t}{1-2 x t+t^{2}}=\sum_{n=0}^{\infty} V_{n}(x) t^{n} \\
G(t, x)=\frac{1+t}{1-2 x t+t^{2}}=\sum_{n=0}^{\infty} W_{n}(x) t^{n} \\
e^{2 x t-t^{2}}=\sum_{n=0}^{\infty} H_{n}(x) \frac{t^{n}}{n !}, \\
(1-t)^{-\alpha-1} \exp \left(-\frac{x t}{1-t}\right)=\sum_{n=0}^{\infty} L_{n}^{\alpha}(x) t^{n},(\alpha>-1) \\
\left(1-2 x t+t^{2}\right)^{-\frac{1}{2}}=\sum_{n=0}^{\infty} P_{n}(x) t^{n}, \\
\frac{1}{\left(1-2 x t+t^{2}\right)^{\lambda}}=\sum_{n=0}^{\infty} C_{n}^{(\lambda)}(x) t^{n},\left(\lambda>-\frac{1}{2}, \lambda \neq 0,|t|<1,|x| \leq 1\right) \\
\frac{2^{\alpha+\beta}}{R(1-t+R)^{\alpha}(1+t+R)^{\beta}}=\sum_{n=0}^{\infty} P_{n}^{(\alpha, \beta)}(x) t^{n}, \\
\left(R=\sqrt{1-2 x t+t^{2}}, \alpha, \beta>-1\right)
\end{gathered}
$$

Explicit expressions for the above special polynomials are as in the following:

$$
\begin{aligned}
V_{n}(x) & ={ }_{2} F_{1}\left(-n, n+1 ; \frac{1}{2} ; \frac{1-x}{2}\right) \\
& =\sum_{l=0}^{n}\left(\begin{array}{c}
2 n-l \\
l
\end{array}\right) 2^{n-l}(x-1)^{n-l},
\end{aligned}
$$




$$
\begin{aligned}
W_{n}(x) & =(2 n+1){ }_{2} F_{1}\left(-n, n+1 ; \frac{3}{2} ; \frac{1-x}{2}\right) \\
& =(2 n+1) \sum_{l=0}^{n} \frac{2^{n-l}}{2 n-2 l+1}\left(\begin{array}{c}
2 n-l \\
l
\end{array}\right)(x-1)^{n-l}, \\
H_{n}(x) & =n ! \sum_{l=0}^{\left[\frac{n}{2}\right]} \frac{(-1)^{l}}{l !(n-2 l) !}(2 x)^{n-2 l}, \\
L_{n}^{\alpha}(x) & =\frac{<\alpha+1>_{n}}{n !} F_{1}(-n, \alpha+1 ; x) \\
& =\sum_{l=0}^{n} \frac{(-1)^{l}\left(\begin{array}{c}
n+\alpha \\
n-l
\end{array}\right)}{l !} x^{l}, \\
P_{n}(x) & ={ }_{2} F_{1}\left(-n, n+1 ; 1 ; \frac{1-x}{2}\right) \\
& =\frac{1}{2^{n}} \sum_{l=0}^{\left[\frac{n}{2}\right]}(-1)^{l}\left(\begin{array}{c}
n \\
l
\end{array}\right)\left(\begin{array}{c}
2 n-2 l \\
n
\end{array}\right) x^{n-2 l}, \\
C_{n}^{(\lambda)}(x) & =\left(\begin{array}{c}
n+2 \lambda-1 \\
n
\end{array}\right) 2 F_{1}\left(-n, n+2 \lambda ; \lambda+\frac{1}{2} ; \frac{1-x}{2}\right) \\
& =\sum_{k=0}^{\left[\frac{n}{2}\right]}(-1)^{k} \frac{\Gamma(n-k+\lambda)}{\Gamma(\lambda) k !(n-2 k) !}(2 x)^{n-2 k}, \\
P_{n}^{(\alpha, \beta)}(x) & =\frac{<\alpha+1>n}{n !}{ }_{2} F_{1}\left(-n, 1+\alpha+\beta+n ; \alpha+1 ; \frac{1-x}{2}\right) \\
& =\sum_{k=0}^{n}\left(\begin{array}{c}
n+\alpha \\
n-k
\end{array}\right)\left(\begin{array}{c}
n+\beta \\
k
\end{array}\right)\left(\frac{x-1}{2}\right)^{k}\left(\frac{x+1}{2}\right)^{n-k} .
\end{aligned}
$$

Next, we state Rodrigues-type formulas for Hermite and generalized Laguerre polynomials and Rodrigues' formulas for Legendre, Gegenbauer and Jacobi polynomials.

$$
\begin{aligned}
& H_{n}(x)=(-1)^{n} e^{x^{2}} \frac{d^{n}}{d x^{n}} e^{-x^{2}}, \\
& L_{n}^{\alpha}(x)=\frac{1}{n !} x^{-\alpha} e^{x} \frac{d^{n}}{d x^{n}}\left(e^{-x} x^{n+\alpha}\right), \\
& P_{n}(x)=\frac{1}{2^{n} n !} \frac{d^{n}}{d x^{n}}\left(x^{2}-1\right)^{n}, \\
& \left(1-x^{2}\right)^{\lambda-\frac{1}{2}} C_{n}^{(\lambda)}(x)=\frac{(-2)^{n}}{n !} \frac{<\lambda>_{n}}{<n+2 \lambda>_{n}} \frac{d^{n}}{d x^{n}}\left(1-x^{2}\right)^{n+\lambda-\frac{1}{2}}, \\
& (1-x)^{\alpha}(1+x)^{\beta} P_{n}^{(\alpha, \beta)}(x)=\frac{(-1)^{n}}{2^{n} n !} \frac{d^{n}}{d x^{n}}(1-x)^{n+\alpha}(1+x)^{n+\beta} .
\end{aligned}
$$

The last thing we want to mention is the orthogonalities with respect to various weight functions enjoyed by Hermite, generalized Laguerre, Legendre, Gegenbauer and Jacobi polynomials.

$$
\begin{aligned}
& \int_{-\infty}^{\infty} e^{-x^{2}} H_{n}(x) H_{m}(x) d x=2^{n} n ! \sqrt{\pi} \delta_{n, m}, \\
& \int_{0}^{\infty} x^{\alpha} e^{-x} L_{n}^{\alpha}(x) L_{m}^{\alpha}(x) d x=\frac{1}{n !} \Gamma(\alpha+n+1) \delta_{n, m}, \\
& \int_{-1}^{1} P_{n}(x) P_{m}(x) d x=\frac{2}{2 n+1} \delta_{n, m}, \\
& \int_{-1}^{1}\left(1-x^{2}\right)^{\lambda-\frac{1}{2}} C_{n}^{(\lambda)}(x) C_{m}^{(\lambda)}(x) d x=\frac{\pi 2^{1-2 \lambda} \Gamma(n+2 \lambda)}{n !(n+\lambda) \Gamma(\lambda)^{2}} \delta_{n, m}
\end{aligned}
$$




$$
\begin{aligned}
& \int_{-1}^{1}(1-x)^{\alpha}(1+x)^{\beta} P_{n}^{(\alpha, \beta)}(x) P_{m}^{(\alpha, \beta)}(x) d x \\
& =\frac{2^{\alpha+\beta+1} \Gamma(n+\alpha+1) \Gamma(n+\beta+1)}{(2 n+\alpha+\beta+1) \Gamma(n+\alpha+\beta+1) \Gamma(n+1)} \delta_{n, m} .
\end{aligned}
$$

For convenience, let us put

$$
\begin{gathered}
\gamma_{n, r}(x)=\sum_{l=0}^{n} \sum_{i_{1}+i_{2}+\cdots+i_{r+1}=l}\left(\begin{array}{c}
r-1+n-l \\
r-1
\end{array}\right) V_{i_{1}}(x) V_{i_{2}}(x) \cdots V_{i_{r+1}}(x),(n \geq 0, r \geq 1), \\
\mathcal{E}_{n, r}(x)=\sum_{l=0}^{n} \sum_{i_{1}+i_{2}+\cdots+i_{r+1}=l}(-1)^{n-l}\left(\begin{array}{c}
r-1+n-l \\
r-1
\end{array}\right) W_{i_{1}}(x) W_{i_{2}}(x) \cdots W_{i_{r+1}}(x),(n \geq 0, r \geq 1) .
\end{gathered}
$$

We observe here that both $\gamma_{n, r}(x)$ and $\mathcal{E}_{n, r}(x)$ have degree $n$.

In this paper, we will consider the connection problem of expressing the sums of finite products in (33) and (34) as linear combinations of $H_{n}(x), L_{n}^{\alpha}(x), P_{n}(x), C_{n}^{(\lambda)}(x)$, and $P_{n}^{(\alpha, \beta)}(x)$. These will be done by performing explicit computations based on Proposition 1 . We observe here that the formulas in Proposition 1 follow from their orthogonalities, Rodrigues' and Rodrigues-type formulas and integration by parts.

Our main results are the following Theorems 1 and 2.

Theorem 1. Let $n, r$ be any integers with $n \geq 0, r \geq 1$. Then we have the following.

$$
\begin{aligned}
\sum_{l=0}^{n} \sum_{i_{1}+i_{2}+\cdots+i_{r+1}=l}\left(\begin{array}{c}
r-1+n-l \\
r-1
\end{array}\right) V_{i_{1}}(x) V_{i_{2}}(x) \cdots V_{i_{r+1}}(x) \\
=\frac{(2 n+2 r) !}{r ! 4^{n+r}\left(n+r-\frac{1}{2}\right)_{n+r}} \sum_{k=0}^{n} \frac{(-2)^{k}}{(n-k) !} \\
\quad \times \sum_{j=0}^{\left[\frac{k}{2}\right]} \frac{{ }_{2} F_{1}\left(2 j-k, \frac{1}{2}-n-r ;-2 n-2 r ; 2\right)}{j ! 4 j(k-2 j) !} H_{n-k}(x) \\
=\frac{1}{r !} \sum_{k=0}^{n} \sum_{l=0}^{k} \frac{(-2)^{n-l}(2 n+2 r-l) !(n+r-l) !}{l !(2 n+2 r-2 l) !(k-l) !} \\
\quad \times{ }_{2} F_{0}(l-k, n-k+\alpha+1 ;-; 1) L_{n-k}^{\alpha}(x) \\
=\frac{(-1)^{n} n !(2 n+2 r) !}{r ! 4^{r}\left(n+r-\frac{1}{2}\right) n+r} \sum_{k=0}^{n} \frac{(-1)^{k}(2 k+1)}{(n-k) !(n+k+1) !} \\
\quad \times{ }_{3} F_{2}\left(k-n, \frac{1}{2}-n-r,-n-k-1 ;-2 n-2 r,-n ; 1\right) P_{k}(x) \\
=\frac{(-1)^{n}(2 n+2 r) ! 4^{\lambda-r} \Gamma(\lambda) \Gamma\left(n+\lambda+\frac{1}{2}\right)}{\sqrt{\pi} r !\left(n+r-\frac{1}{2}\right)_{n+r}} \frac{(-1)^{k}(k+\lambda)}{\Gamma(n+k+2 \lambda+1)(n-k) !} \\
\quad \times{ }_{3} F_{2}\left(k-n, \frac{1}{2}-n-r,-n-k-2 \lambda ;-2 n-2 r,-n-\lambda+\frac{1}{2} ; 1\right) C_{k}^{(\lambda)}(x) \\
=\frac{(-1)^{n}(2 n+2 r) ! \Gamma(n+\alpha+1)}{r ! 4^{r}\left(n+r-\frac{1}{2}\right)_{n+r} \sum_{k=0} \frac{(-1)^{k}(2 k+\alpha+\beta+1) \Gamma(k+\alpha+\beta+1)}{(n-k) !(\alpha+k+1) \Gamma(n+k+\alpha+\beta+2)}} \\
\quad \times{ }_{3} F_{2}\left(k-n, \frac{1}{2}-n-r,-n-k-\alpha-\beta-1 ;-2 n-2 r,-n-\alpha ; 1\right) P_{k}^{(\alpha, \beta)}(x) .
\end{aligned}
$$


Theorem 2. Let $n, r$ be any integers with $n \geq 0, r \geq 1$. Then we have the following.

$$
\begin{aligned}
& \sum_{l=0}^{n} \sum_{i_{1}+i_{2}+\cdots+i_{r+1}=l}(-1)^{n-l}\left(\begin{array}{c}
r-1+n-l \\
r-1
\end{array}\right) W_{i_{1}}(x) W_{i_{2}}(x) \cdots W_{i_{r+1}}(x) \\
& =\frac{(2 n+1)(2 n+2 r) !}{r ! 2^{2 n+2 r+1}\left(n+r+\frac{1}{2}\right)_{n+r+1}} \sum_{k=0}^{n} \frac{(-2)^{k}}{(n-k) !} \\
& \times \sum_{j=0}^{\left[\frac{k}{2}\right]} \frac{{ }_{2} F_{1}\left(2 j-k,-n-r-\frac{1}{2} ;-2 n-2 r ; 2\right)}{j ! 4 j(k-2 j) !} H_{n-k}(x) \\
& =\frac{(2 n+1)}{r !} \sum_{k=0}^{n} \sum_{l=0}^{k} \frac{(-2)^{n-l}(2 n+2 r-l) !(n+r-l) !}{l !(2 n+2 r-2 l+1) !(k-l) !} \\
& \times{ }_{2} F_{0}(l-k, n-k+\alpha+1 ;-; 1) L_{n-k}^{\alpha}(x) \\
& =\frac{(-1)^{n} n !(2 n+1)(2 n+2 r) !}{r ! 2^{2 r+1}\left(n+r+\frac{1}{2}\right)_{n+r+1}} \sum_{k=0}^{n} \frac{(-1)^{k}(2 k+1)}{(n-k) !(n+k+1) !} \\
& \times{ }_{3} F_{2}\left(k-n,-n-r-\frac{1}{2},-n-k-1 ;-2 n-2 r,-n ; 1\right) P_{k}(x) \\
& =\frac{(-1)^{n}(2 n+2 r) ! 2^{2 \lambda-2 r-1}(2 n+1) \Gamma(\lambda) \Gamma\left(n+\lambda+\frac{1}{2}\right)}{\sqrt{\pi} r !\left(n+r+\frac{1}{2}\right)_{n+r+1}} \\
& \times \sum_{k=0}^{n} \frac{(-1)^{k}(k+\lambda)}{\Gamma(n+k+2 \lambda+1)(n-k) !} \\
& \times{ }_{3} F_{2}\left(k-n,-n-r-\frac{1}{2},-n-k-2 \lambda ;-2 n-2 r,-n-\lambda+\frac{1}{2} ; 1\right) C_{k}^{(\lambda)}(x) \\
& =\frac{(-1)^{n}(2 n+2 r) !(2 n+1) \Gamma(n+\alpha+1)}{r ! 2^{2 r+1}\left(n+r+\frac{1}{2}\right)_{n+r+1}} \\
& \times \sum_{k=0}^{n} \frac{(-1)^{k}(2 k+\alpha+\beta+1) \Gamma(k+\alpha+\beta+1)}{(n-k) ! \Gamma(\alpha+k+1) \Gamma(n+k+\alpha+\beta+2)} \\
& \times{ }_{3} F_{2}\left(k-n,-n-r-\frac{1}{2},-n-k-\alpha-\beta-1 ;-2 n-2 r,-n-\alpha ; 1\right) P_{k}^{(\alpha, \beta)}(x) .
\end{aligned}
$$

Before closing the section, we are going to mention some of previous results on the related connection problems. The papers [14-16] treat the connection problem of expressing sums of finite products of Bernoulli, Euler and Genocchi polynomials in terms of Bernoulli polynomials. In fact, they were carried out by deriving Fourier series expansions for the functions closely related to those sums of finite products. Moreover, the same was done for the sums of finite products of Chebyshev polynomials of the second kind and of Fibonacci polynomials in [17].

Along the same line as the present paper, sums of finite products of Chebyshev polynomials of the second kind and Fibonacci polynomials were expressed in [18] as linear combinations of the orthogonal polynomials $H_{n}(x), L_{n}^{\alpha}(x), P_{n}(x), C_{n}^{(\lambda)}(x)$, and $P_{n}^{(\alpha, \beta)}(x)$. Also, the connection problem of expressing in terms of all kinds of Chebyshev polynomials were done for sums of finite products of Chebyshev polynomials of the second, third and fourth kinds and of Fibonacci, Legendre and Laguerre polynomials in [19-21].

Finally, we let the reader refer to $[22,23]$ for some applications of Chebyshev polynomials.

\section{Proof of Theorem 1}

First, we will state Propositions 1 and 2 that will be needed in showing Theorems 1 and 2. 
The results in (a), (b), (c), (d) and (e) in Proposition 1 follow respectively from (3.7) of [4], (2.3) of [4] (see also (2.4) of [2]), (2.3) of [5], (2.3) of [3] and (2.7) of [7]. They can be derived from their orthogonalities in (28)-(32), Rodrigues-type and Rodrigues' formulas in (23)-(27) and integration by parts.

Proposition 1. Let $q(x) \in \mathbb{R}[x]$ be a polynomial of degree $n$. Then we have the following.

(a) $q(x)=\sum_{k=0}^{n} C_{k, 1} H_{k}(x)$, where

$$
C_{k, 1}=\frac{(-1)^{k}}{2^{k} k ! \sqrt{\pi}} \int_{-\infty}^{\infty} q(x) \frac{d^{k}}{d x^{k}} e^{-x^{2}} d x,
$$

(b) $q(x)=\sum_{k=0}^{n} C_{k, 2} L_{k}^{\alpha}(x)$, where

$$
C_{k, 2}=\frac{1}{\Gamma(\alpha+k+1)} \int_{0}^{\infty} q(x) \frac{d^{k}}{d x^{k}}\left(e^{-x} x^{k+\alpha}\right) d x,
$$

(c) $q(x)=\sum_{k=0}^{n} C_{k, 3} P_{k}(x)$, where

$$
C_{k, 3}=\frac{2 k+1}{2^{k+1} k !} \int_{-1}^{1} q(x) \frac{d^{k}}{d x^{k}}\left(x^{2}-1\right)^{k} d x,
$$

(d) $q(x)=\sum_{k=0}^{n} C_{k, 4} C_{k}^{(\lambda)}(x)$, where

$$
C_{k, 4}=\frac{(k+\lambda) \Gamma(\lambda)}{(-2)^{k} \sqrt{\pi} \Gamma\left(k+\lambda+\frac{1}{2}\right)} \int_{-1}^{1} q(x) \frac{d^{k}}{d x^{k}}\left(1-x^{2}\right)^{k+\lambda-\frac{1}{2}} d x,
$$

(e) $q(x)=\sum_{k=0}^{n} C_{k, 5} P_{n}^{(\alpha, \beta)}(x)$, where

$$
\begin{aligned}
C_{k, 5} & =\frac{(-1)^{k}(2 k+\alpha+\beta+1) \Gamma(k+\alpha+\beta+1)}{2^{\alpha+\beta+k+1} \Gamma(\alpha+k+1) \Gamma(\beta+k+1)} \\
& \times \int_{-1}^{1} q(x) \frac{d^{k}}{d x^{k}}(1-x)^{k+\alpha}(1+x)^{k+\beta} d x .
\end{aligned}
$$

The following proposition will be used in showing Theorems 1 and 2. In fact, (a) is needed for (35) and (40), (b) for (39) and (44), and (b) or (c) for (37), (38), (42) and (43).

Proposition 2. The following holds true.

(a) For any nonnegative integer $m$,

$$
\int_{-\infty}^{\infty} x^{m} e^{-x^{2}} d x= \begin{cases}0, & \text { if } m \equiv 1(\bmod 2), \\ \frac{m ! \sqrt{\pi}}{\left(\frac{m}{2}\right) ! 2^{m}}, & \text { if } m \equiv 0(\bmod 2),\end{cases}
$$

(b) For any real numbers $r, s>-1$, we have

$$
\int_{-1}^{1}(1-x)^{r}(1+x)^{s} d x=2^{r+s+1} \frac{\Gamma(r+1) \Gamma(s+1)}{\Gamma(r+s+2)},
$$

(c) For any real numbers $r$, w with $r+s>-1, s>-1$, we have

$$
\int_{-1}^{1}(1-x)^{r}\left(1-x^{2}\right)^{s} d x=2^{r+2 s+1} \frac{\Gamma(r+s+1) \Gamma(s+1)}{\Gamma(r+2 s+2)} .
$$




\section{Proof.}

(a) This is an easy exercise.

(c) This follows from (b) with $r$ replaced by $r+s$.

(b) This follows from the change of variable $1+x=2 y$ and (5).

The following lemma can be obtained by differentiating (9), as was shown in [24].

Lemma 1. Let $n, r$ be integers with $n \geq 0, r \geq 1$. Then we have the following identity.

$$
\sum_{l=0}^{n} \sum_{i_{1}+i_{2}+\cdots+i_{r+1}=l}\left(\begin{array}{c}
r-1+n-l \\
r-1
\end{array}\right) V_{i_{1}}(x) V_{i_{2}}(x) \cdots V_{i_{r+1}}(x)=\frac{1}{2^{r} r !} V_{n+r}^{(r)}(x)
$$

where the inner sum runs over all nonnegative integers $i_{1}, i_{2}, \cdots i_{r+1}$, with $i_{1}+i_{2}+\cdots+i_{r+1}=l$.

From (16), we see that the $r$ th derivative of $V_{n}(x)$ is given by

$$
V_{n}^{(r)}(x)=\sum_{l=0}^{n-r}\left(\begin{array}{c}
2 n-l \\
l
\end{array}\right) 2^{n-l}(n-l)_{r}(x-1)^{n-l-r} .
$$

Especially, we have

$$
V_{n+r}^{(r+k)}(x)=\sum_{l=0}^{n-k}\left(\begin{array}{c}
2 n+2 r-l \\
l
\end{array}\right) 2^{n+r-l}(n+r-l)_{r+k}(x-1)^{n-k-l} .
$$

Now, we are ready to prove Theorem 1. As (38) and (39) can be shown similarly to (43) and (44) in the next section, we will show only (35), (36) and (37). With $\gamma_{n, r}(x)$ as in (33), we let

$$
\gamma_{n, r}(x)=\sum_{k=0}^{n} C_{k, 1} H_{k}(x) .
$$

Then, from (a) of Proposition 1, (45), (47), and integration by parts $k$ times, we obtain

$$
\begin{aligned}
C_{k, 1}= & \frac{(-1)^{k}}{2^{k} k ! \sqrt{\pi}} \int_{-\infty}^{\infty} \gamma_{n, r}(x) \frac{d^{k}}{d x^{k}} e^{-x^{2}} d x \\
= & \frac{(-1)^{k}}{2^{k+r} k ! r ! \sqrt{\pi}} \int_{-\infty}^{\infty} V_{n+r}^{(r)}(x) \frac{d^{k}}{d x^{k}} e^{-x^{2}} d x \\
= & \frac{1}{2^{k+r} k ! r ! \sqrt{\pi}} \int_{-\infty}^{\infty} V_{n+r}^{(r+k)}(x) e^{-x^{2}} d x \\
= & \frac{1}{2^{k+r} k ! r ! \sqrt{\pi}} \sum_{l=0}^{n-k}\left(\begin{array}{c}
2 n+2 r-l \\
l
\end{array}\right) 2^{n+r-l}(n+r-l)_{r+k} \\
& \times \int_{-\infty}^{\infty}(x-1)^{n-k-l} e^{-x^{2}} d x .
\end{aligned}
$$


Before proceeding further, by making use of (a) in Proposition 2, we note that

$$
\begin{aligned}
& \int_{-\infty}^{\infty}(x-1)^{m} e^{-x^{2}} d x \\
= & \sum_{s=0}^{m}\left(\begin{array}{c}
m \\
s
\end{array}\right)(-1)^{m-s} \int_{-\infty}^{\infty} x^{s} e^{-x^{2}} d x \\
= & \sum_{\substack{0 \leq s \leq m \\
s \equiv 0(\bmod 2)}}\left(\begin{array}{c}
m \\
s
\end{array}\right)(-1)^{m-s} \frac{s ! \sqrt{\pi}}{\left(\frac{s}{2}\right) ! 2^{s}} \\
= & (-1)^{m} \sqrt{\pi} \sum_{j=0}^{\left[\frac{m}{2}\right]}\left(\begin{array}{c}
m \\
2 j
\end{array}\right) \frac{(2 j) !}{j ! 2^{2 j}},(m \geq 0) .
\end{aligned}
$$

From (48)-(50), and after simplifications, we have

$$
\begin{aligned}
\gamma_{n, r}(x) & =\frac{1}{r !} \sum_{k=0}^{n} \frac{(-2)^{k}}{(n-k) !} \sum_{l=0}^{k} \sum_{j=0}^{\left[\frac{k-l}{2}\right]} \frac{\left(-\frac{1}{2}\right)^{l}(2 n+2 r-l) !(n+r-l) !}{l !(2 n+2 r-2 l) !(k-l-2 j) ! j ! 4^{j}} H_{n-k}(x) \\
& =\frac{1}{r !} \sum_{k=0}^{n} \frac{(-2)^{k}}{(n-k) !} \sum_{j=0}^{\left[\frac{k}{2}\right]} \frac{1}{j ! 4^{j}} \sum_{l=0}^{k-2 j} \frac{\left(-\frac{1}{2}\right)^{l}(2 n+2 r-l) !(n+r-l) !}{l !(2 n+2 r-2 l) !(k-l-2 j) !} H_{n-k}(x) \\
& =\frac{(2 n+2 r) !}{r ! 4^{n+r}<\frac{1}{2}>_{n+r}} \sum_{k=0}^{n} \frac{(-2)^{k}}{(n-k) !} \sum_{j=0}^{\left[\frac{k}{2}\right]} \frac{1}{j ! 4^{j}(k-2 j) !} \\
& \times \sum_{l=0}^{k-2 j} \frac{2^{l}<2 j-k>_{l}<\frac{1}{2}-n-r>_{l}}{l !<-2 n-2 r>_{l}} H_{n-k}(x) \\
& =\frac{(2 n+2 r) !}{r ! 4^{n+r}\left(n+r-\frac{1}{2}\right)_{n+r}} \sum_{k=0}^{n} \frac{(-2)^{k}}{(n-k) !} \\
& \times \sum_{j=0}^{\left[\frac{k}{2}\right]} \frac{{ }_{2} F_{1}\left(2 j-k, \frac{1}{2}-n-r ;-2 n-2 r ; 2\right)}{j ! 4^{j}(k-2 j) !} H_{n-k}(x) .
\end{aligned}
$$

This shows (35) of Theorem 1.

Next, we let

$$
\gamma_{n, r}(x)=\sum_{k=0}^{n} C_{k, 2} L_{k}^{\alpha}(x) .
$$

Then, from (b) of Proposition 1, (45), (47) and integration by parts $k$ times, we get 


$$
\begin{aligned}
C_{k, 2}= & \frac{1}{2^{r} r ! \Gamma(\alpha+k+1)} \int_{0}^{\infty} V_{n+r}^{(r)}(x) \frac{d^{k}}{d x^{k}}\left(e^{-x} x^{k+\alpha}\right) d x \\
= & \frac{(-1)^{k}}{2^{r} r ! \Gamma(\alpha+k+1)} \int_{0}^{\infty} V_{n+r}^{(r+k)}(x) e^{-x} x^{k+\alpha} d x \\
= & \frac{(-1)^{k}}{2^{r} r ! \Gamma(\alpha+k+1)} \sum_{l=0}^{n-k}\left(\begin{array}{c}
2 n+2 r-l \\
l
\end{array}\right) 2^{n+r-l}(n+r-l)_{r+k} \\
& \times \int_{0}^{\infty}(x-1)^{n-k-l} e^{-x} x^{k+\alpha} d x \\
= & \frac{(-1)^{k}}{2^{r} r ! \Gamma(\alpha+k+1)} \sum_{l=0}^{n-k}\left(\begin{array}{c}
2 n+2 r-l \\
l
\end{array}\right) 2^{n+r-l}(n+r-l)_{r+k} \\
& \times \sum_{s=0}^{n-k-l}\left(\begin{array}{c}
n-k-l \\
s
\end{array}\right)(-1)^{n-k-l-s} \Gamma(s+k+\alpha+1) \\
= & \frac{(-1)^{k}}{2^{r} r !} \sum_{l=0}^{n-k}\left(\begin{array}{c}
2 n+2 r-l \\
l
\end{array}\right) 2^{n+r-l}(n+r-l)_{r+k} \\
& \times \sum_{s=0}^{n-k-l}\left(\begin{array}{c}
n-k-l \\
s
\end{array}\right)(-1)^{n-k-l-s}<k+\alpha+1>_{s} \\
= & \frac{1}{r !} \sum_{l=0}^{n-k} \frac{(2 n+2 r-l) !(-2)^{n-l}(n+r-l) !}{l !(2 n+2 r-2 l) !(n-k-l) !} \\
& \times \sum_{s=0}^{n-k-l} \frac{1}{s !}<k+l-n>s<k+\alpha+1>>_{s} \\
= & \frac{1}{r !} \sum_{l=0}^{n-k} \frac{(2 n+2 r-l) !(-2)^{n-l}(n+r-l) !}{l !(2 n+2 r-2 l) !(n-k-l) !} \\
& \times{ }_{2} F_{0}(k+l-n, k+\alpha+1 ;-; 1) .
\end{aligned}
$$

Combining (52)-(53), we finally have

$$
\begin{aligned}
\gamma_{n, r}(x)= & \frac{1}{r !} \sum_{k=0}^{n} \sum_{l=0}^{k} \frac{(2 n+2 r-l) !(-2)^{n-l}(n+r-l) !}{l !(2 n+2 r-2 l) !(k-l) !} \\
& \times{ }_{2} F_{0}(l-k, n-k+\alpha+1 ;-; 1) L_{n-k}^{\alpha}(x) .
\end{aligned}
$$

This completes the proof for (36) of Theorem 1.

Finally, let us put

$$
\gamma_{n, r}(x)=\sum_{k=0}^{n} C_{k, 3} P_{k}(x) .
$$

Then, from (c) of Proposition 1, (45), (47) and integration by parts $k$ times, we have

$$
\begin{aligned}
C_{k, 3}= & \frac{2 k+1(-1)^{k}}{2^{k+r+1} k ! r !} \int_{-1}^{1} V_{n+r}^{(r+k)}(x)\left(x^{2}-1\right)^{k} d x \\
= & \frac{(2 k+1)(-1)^{k}}{2^{k+r+1} k ! r !} \sum_{l=0}^{n-k}\left(\begin{array}{c}
2 n+2 r-l \\
l
\end{array}\right) 2^{n+r-l}(n+r-l)_{r+k} \\
& \times \int_{-1}^{1}(x-1)^{n-k-l}\left(x^{2}-1\right)^{k} d x .
\end{aligned}
$$


By making use of (c) in Proposition 2 and after simplifications, from (56) we obtain

$$
\begin{aligned}
C_{k, 3}= & \frac{(-1)^{n+k}(2 k+1)}{r !} \sum_{l=0}^{n-k} \\
& \times \frac{(-1)^{l} 4^{n-l}(2 n+2 r-l) !(n+r-l) !(n-l) !}{l !(2 n+2 r-2 l) !(n-k-l) !(n+k-l+1) !} \\
= & \frac{(-1)^{n}(2 n+2 r) ! n !}{r ! 4^{r}\left(n+r-\frac{1}{2}\right)_{n+r}} \frac{(-1)^{k}(2 k+1)}{(n-k) !(n+k+1) !} \\
& \times \sum_{l=0}^{n-k} \frac{<k-n>_{l}<\frac{1}{2}-n-r>_{l}<-n-k-1>_{l}}{l !<-2 n-2 r>_{l}<-n>_{l}} \\
= & \frac{(-1)^{n}(2 n+2 r) ! n !}{r ! 4^{r}\left(n+r-\frac{1}{2}\right)_{n+r}} \frac{(-1)^{k}(2 k+1)}{(n-k) !(n+k+1) !} \\
& \times{ }_{3} F_{2}\left(k-n, \frac{1}{2}-n-r,-n-k-1 ;-2 n-2 r,-n ; 1\right) .
\end{aligned}
$$

From (55) and (57), we get

$$
\begin{aligned}
\gamma_{n, r}(x)= & \frac{(-1)^{n}(2 n+2 r) ! n !}{r ! 4^{r}\left(n+r-\frac{1}{2}\right)_{n+r}} \sum_{k=0}^{n} \frac{(-1)^{k}(2 k+1)}{(n-k) !(n+k+1) !} \\
& \times{ }_{3} F_{2}\left(k-n, \frac{1}{2}-n-r,-n-k-1 ;-2 n-2 r,-n ; 1\right) P_{k}(x) .
\end{aligned}
$$

This proves (37) of Theorem 1.

\section{Proof of Theorem 2}

Here we will show only (43) and (44) in Theorem 2, as (40)-(42) can be shown analogously to the proofs for (35)-(37), respectively. The following can be derived by differentiating the Equation (10) and is stated in [24].

Lemma 2. Let $n, r$ be integers with $n \geq 0, r \geq 1$. Then we have the following identity.

$$
\sum_{l=0}^{n} \sum_{i_{1}+i_{2}+\cdots+i_{r+1}=l}(-1)^{n-l}\left(\begin{array}{c}
r-1+n-l \\
r-1
\end{array}\right) W_{i_{1}}(x) W_{i_{2}}(x) \cdots W_{i_{r+1}}(x)=\frac{1}{2^{r} r !} W_{n+r}^{(r)}(x),
$$

where the inner sum runs over all nonnegative integers $i_{1}, i_{2}, \cdots, i_{r+1}$, with $i_{1}+i_{2}+\cdots+i_{r+1}=l$.

From (17), the $r$ th derivative of $W_{n}(x)$ is given by

$$
W_{n}^{(r)}(x)=(2 n+1) \sum_{l=0}^{n-r} \frac{2^{n-l}}{2 n+1-2 l}\left(\begin{array}{c}
2 n-l \\
l
\end{array}\right)(n-l)_{r}(x-1)^{n-l-r} .
$$

In particular, we have

$$
W_{n+r}^{(r+k)}(x)=(2 n+1) \sum_{l=0}^{n-k} \frac{2^{n+r-l}}{2 n+2 r+1-2 l}\left(\begin{array}{c}
2 n+2 r-l \\
l
\end{array}\right)(n+r-l)_{r+k}(x-1)^{n-k-l} .
$$

With $\mathcal{E}_{n, r}(x)$ as in (34), we let

$$
\mathcal{E}_{n, r}(x)=\sum_{k=0}^{n} C_{k, 4} C_{k}^{(\alpha)}(x)
$$


Then, from (d) of Proposition 1, (59), (61) and integration by parts $k$ times, we get

$$
\begin{aligned}
C_{k, 4} & =\frac{(k+\lambda) \Gamma(\lambda)}{2^{k+r} r ! \sqrt{\pi} \Gamma\left(k+\lambda+\frac{1}{2}\right)} \\
& \times \int_{-1}^{1} W_{n+r}^{(r+k)}(x)\left(1-x^{2}\right)^{k+\lambda-\frac{1}{2}} d x \\
& =\frac{(k+\lambda) \Gamma(\lambda)(2 n+1)}{2^{k+r} r ! \sqrt{\pi} \Gamma\left(k+\lambda+\frac{1}{2}\right)} \\
& \times \sum_{l=0}^{n-k} \frac{2^{n+r-l}}{2 n+2 r+1-2 l}\left(\begin{array}{c}
2 n+2 r-l \\
l
\end{array}\right)(n+r-l)_{r+k} \\
& \times \int_{-1}^{1}(x-1)^{n-k-l}\left(1-x^{2}\right)^{k+\lambda-\frac{1}{2}} d x \\
& =\frac{(k+\lambda) \Gamma(\lambda)(2 n+1)(-2)^{n-k}}{r ! \sqrt{\pi} \Gamma\left(k+\lambda+\frac{1}{2}\right)} \\
& \times \sum_{l=0}^{n-k} \frac{\left(-\frac{1}{2}\right)^{l}(2 n+2 r-l) !(n+r-l) !}{(2 n+2 r-2 l+1) l !(2 n+2 r-2 l) !(n-k-l) !} \\
& \times \int_{-1}^{1}(1-x)^{n-k-l}\left(1-x^{2}\right)^{k+\lambda-\frac{1}{2}} d x .
\end{aligned}
$$

Invoking (c) of Proposition 2 and after simplifications, from (63) we obtain

$$
\begin{aligned}
C_{k, 4} & =\frac{(-1)^{n-k}(k+\lambda) \Gamma(\lambda)(2 n+1) 2^{2 n+2 \lambda+1} \Gamma\left(n+\lambda+\frac{1}{2}\right)(2 n+2 r) !}{\Gamma(n+k+2 \lambda+1)(n-k) ! r ! \sqrt{\pi}} \\
& \times \sum_{l=0}^{n-k} \frac{\left(-\frac{1}{4}\right)^{l}(2 n+2 r-l) !(n+r-l+1) !(n-k) !(n+k+2 \lambda)_{l}}{l !(2 n+2 r) !(2 n+2 r-2 l+2) !(n-k-l) !\left(n+\lambda-\frac{1}{2}\right)_{l}} \\
& =\frac{(-1)^{k}(k+\lambda) \Gamma(\lambda)(2 n+1) 2^{2 \lambda-2 r-1}(-1)^{n} \Gamma\left(n+\lambda+\frac{1}{2}\right)(2 n+2 r) !}{\Gamma(n+k+2 \lambda+1)(n-k) ! r ! \sqrt{\pi}\left(n+r+\frac{1}{2}\right)_{n+r+1}} \\
& \times \sum_{l=0}^{n-k} \frac{<k-n>_{l}<-n-r-\frac{1}{2}>_{l}<-n-k-2 \lambda>_{l}}{l !<-2 n-2 r>_{l}<-n-\lambda+\frac{1}{2}>_{l}} \\
& =\frac{(-1)^{k}(k+\lambda) \Gamma(\lambda)(2 n+1) 2^{2 \lambda-2 r-1}(-1)^{n} \Gamma\left(n+\lambda+\frac{1}{2}\right)(2 n+2 r) !}{\Gamma(n+k+2 \lambda+1)(n-k) ! r ! \sqrt{\pi}\left(n+r+\frac{1}{2}\right)_{n+r+1}} \\
& \times{ }_{3} F_{2}\left(k-n,-n-r-\frac{1}{2},-n-k-2 \lambda ;-2 n-2 r,-n-\lambda+\frac{1}{2} ; 1\right) .
\end{aligned}
$$

From (62) and (64), we have

$$
\begin{aligned}
\mathcal{E}_{n, r}(x) & =\frac{\Gamma(\lambda)(2 n+1) 2^{2 \lambda-2 r-1}(-1)^{n} \Gamma\left(n+\lambda+\frac{1}{2}\right)(2 n+2 r) !}{r ! \sqrt{\pi}\left(n+r+\frac{1}{2}\right)_{n+r+1}} \\
& \times \sum_{k=0}^{n} \frac{(-1)^{k}(k+\lambda)}{\Gamma(n+k+2 \lambda+1)(n-k) !} \\
& \times{ }_{3} F_{2}\left(k-n,-n-r-\frac{1}{2},-n-k-2 \lambda ;-2 n-2 r,-n-\lambda+\frac{1}{2} ; 1\right) C_{k}^{(\alpha)}(x) .
\end{aligned}
$$

This shows (43) of Theorem 2.

Next, we let

$$
\mathcal{E}_{n, r}(x)=\sum_{k=0}^{n} C_{k, 5} P_{n}^{(\alpha, \beta)}(x) .
$$


Then, from (e) of Proposition 1, and (59), (61), and integrating by parts $k$ times, we obtain

$$
\begin{aligned}
C_{k, 5}= & \frac{(2 k+\alpha+\beta+1) \Gamma(k+\alpha+\beta+1)}{2^{\alpha+\beta+k+r+1} r ! \Gamma(\alpha+k+1) \Gamma(\beta+k+1)} \\
& \times \int_{-1}^{1} W_{n+r}^{(r+k)}(x)(1-x)^{k+\alpha}(1+x)^{k+\beta} d x \\
= & \frac{(2 k+\alpha+\beta+1) \Gamma(k+\alpha+\beta+1)(2 n+1)}{2^{\alpha+\beta+k+r+1} r ! \Gamma(\alpha+k+1) \Gamma(\beta+k+1)} \\
& \times \sum_{l=0}^{n-k} \frac{2^{n+r-l}}{2 n+2 r-2 l+1}\left(\begin{array}{c}
2 n+2 r-l \\
l
\end{array}\right)(n+r-l)_{r+k}(-1)^{n-k-l} \\
& \times \int_{-1}^{1}(1-x)^{n+\alpha-l}(1+x)^{k+\beta} d x .
\end{aligned}
$$

By exploiting (b) in Proposition 2 and after simplifications, from (67) we get

$$
\begin{aligned}
C_{k, 5} & =\frac{(-1)^{n-k}(2 k+\alpha+\beta+1) \Gamma(k+\alpha+\beta+1) 2^{2 n+1}(2 n+1) \Gamma(n+\alpha+1)}{\Gamma(\alpha+k+1) \Gamma(n+k+\alpha+\beta+2) r !} \\
& \times \sum_{l=0}^{n-k} \frac{\left(-\frac{1}{4}\right)^{l}(2 n+2 r-l) !(n+r-l+1) !(n+k+\alpha+\beta+1)_{l}}{l !(2 n+2 r-2 l+2) !(n-k-l) !(n+\alpha)_{l}} \\
& =\frac{(-1)^{n-k}(2 k+\alpha+\beta+1) \Gamma(k+\alpha+\beta+1)(2 n+1) \Gamma(n+\alpha+1)(2 n+2 r) !}{\Gamma(\alpha+k+1) \Gamma(n+k+\alpha+\beta+2)(n-k) ! r ! 2^{2 r+1}\left(n+r+\frac{1}{2}\right)_{n+r+1}} \\
& \times \sum_{l=0}^{n-k} \frac{<k-n>_{l}<-n-r-\frac{1}{2}>_{l}<-n-k-\alpha-\beta-1>_{l}}{l !<-2 n-2 r>_{l}<-n-\alpha>l} \\
& =\frac{(-1)^{n-k}(2 k+\alpha+\beta+1) \Gamma(k+\alpha+\beta+1)(2 n+1) \Gamma(n+\alpha+1)(2 n+2 r) !}{\Gamma(\alpha+k+1) \Gamma(n+k+\alpha+\beta+2)(n-k) ! r ! 2^{2 r+1}\left(n+r+\frac{1}{2}\right)_{n+r+1}} \\
& \times{ }_{3} F_{2}\left(k-n,-n-r-\frac{1}{2},-n-k-\alpha-\beta-1 ;-2 n-2 r,-n-\alpha ; 1\right) .
\end{aligned}
$$

Thus, from (66) and (68), we have

$$
\begin{aligned}
& \mathcal{E}_{n, r}(x)=\frac{(-1)^{n}(2 n+1) \Gamma(n+\alpha+1)(2 n+2 r) !}{r ! 2^{2 r+1}\left(n+r+\frac{1}{2}\right)_{n+r+1}} \\
& \quad \times \sum_{k=0}^{n} \frac{(-1)^{k}(2 k+\alpha+\beta+1) \Gamma(k+\alpha+\beta+1)}{\Gamma(\alpha+k+1) \Gamma(n+k+\alpha+\beta+2)(n-k) !} \\
& \quad \times{ }_{3} F_{2}\left(k-n,-n-r-\frac{1}{2},-n-k-\alpha-\beta-1 ;-2 n-2 r,-n-\alpha ; 1\right) P_{n}^{(\alpha, \beta)}(x) .
\end{aligned}
$$

\section{Conclusions}

In this paper, we considered sums of finite products of Chebyshev polynomials of the third and fourth kinds and expressed each of them in terms of five orthogonal polynomials. Indeed, by explicit computations we expressed each of them as linear combinations of Hermite, generalized Laguerre, Legendre, Gegenbauer and Jacobi polynomials which involve some terminating hypergeometric functions. This can be viewed as a generalization of the classical linearization problem. In general, the linearization problem deals with determining the coefficients in the expansion of the products of two polynomials $a_{m}(x)$ and $b_{n}(x)$ in terms of an arbitrary polynomial sequence $\left\{p_{k}(x)\right\}_{k \geq 0}$ :

$$
a_{m}(x) b_{n}(x)=\sum_{k=0}^{m+n} c_{m n}(k) p_{k}(x) .
$$


Those sums of finite products were also represented by all kinds of Chebyshev polynomials in [20]. In addition, the same had been done for sums of finite products of Chebyshev polynomials of the first and second kinds, Fibonacci polynomials, Legendre polynomials, Laguerre polynomials and Lucas polynomials.

Author Contributions: T.K. and D.S.K. conceived the framework and structured the whole paper; T.K. wrote the paper; J.K. typed; D.V.D. checked for typos; D.S.K.completed the revision of the article.

Funding: This work was supported by the National Research Foundation of Korea (NRF) grant funded by the Korea government (MEST) (No. 2017R1E1A1A03070882).

Acknowledgments: We would like to thank the referees for their valuable comments and suggestions.

Conflicts of Interest: The authors declare no conflict of interest.

\section{References}

1. Kim, D.S.; Dolgy, D.V.; Kim, T.; Rim, S.H. Identities involving Bernoulli and Euler polynomials arising from Chebyshev polynomials. Proc. Jangjeon Math. Soc. 2012, 15, 361-370.

2. Kim, D.S.; Kim, T.; Dolgy, D.V. Some identities on Laguerre polynomials in connection with Bernoulli and Euler numbers. Discret. Dyn. Nat. Soc. 2012, 2012, 619197. [CrossRef]

3. Kim, D.S.; Kim, T.; Rim, S.-H. Some identities involving Gegenbauer polynomials. Adv. Differ. Equ. 2012, 2012, 219. [CrossRef]

4. Kim, D.S.; Kim, T.; Rim, S.-H.; Lee, S.H. Hermite polynomials and their applications associated with Bernoulli and Euler numbers. Discret. Dyn. Nat. Soc. 2012, 2012, 974632. [CrossRef]

5. Kim, D.S.; Rim, S.-H.; Kim, T. Some identities on Bernoulli and Euler polynomials arising from orthogonality of Legendre polynomials. J. Inequal. Appl. 2012, 2012, 227. [CrossRef]

6. Kim, T.; Kim, D.S. Extended Laguerre polynomials associated with Hermite, Bernoulli, and Euler numbers and polynomials. Abstr. Appl. Anal. 2012, 2012, 957350. [CrossRef]

7. Kim, T.; Kim, D.S.; Dolgy, D.V. Some identities on Bernoulli and Hermite polynomials associated with Jacobi polynomials. Discret. Dyn. Nat. Soc. 2012, 2012, 584643. [CrossRef]

8. Kim, T.; Kim, D.S. Identities for degenerate Bernoulli polynomials and Korobov polynomials of the first kind. Sci. China Math. 2018. [CrossRef]

9. Kim, T.; Kim, D.S.; Kwon, J.; Gang, -W. J. Sums of finite products of Legendre and Laguerre polynomials by Chebyshev polynomials. Adv. Stud. Contemp. Math. (Kyungshang) 2018, 28, 551-565.

10. Andrews, G.E.; Askey, R.; Roy, R. Special Functions; Encyclopedia of Mathematics and its Applications 71; Cambridge University Press: Cambridge, UK, 1999.

11. Beals, R.; Wong, R. Special Functions and Orthogonal Polynomials; Cambridge Studies in Advanced Mathematics 153; Cambridge University Press: Cambridge, UK, 2016.

12. Wang, Z.X.; Guo, D.R. Special Functions; Translated by Guo, D.R., Xia, X.J.; World Scientific Publishing Co., Inc.: Teaneck, NJ, USA, 1989.

13. Mason, J.C.; Handscomb, D.C. Chebyshev Polynomials; Chapman \& Hall/CRC: Boca Raton, FL, USA, 2003.

14. Agarwal, R.P.; San Kim, D.; Kim, T.; Kwon, J. Sums of finite products of Bernoulli functions. Adv. Differ. Equ. 2017, 2017, 237. [CrossRef]

15. Kim, T.; San Kim, D.; Jang, G.W.; Kwon, J. Sums of finite products of Euler functions. In Advances in Real and Complex Analysis with Applications; Trends in Mathematics; Birkhäuser: Singapore, 2017; pp. 243-260.

16. Kim, T.; Kim, D.S.; Jang, L.C.; Jang, G.-W. Sums of finite products of Genocchi functions. Adv. Differ. Equ. 2017, 2017, 268. [CrossRef]

17. Kim, T.; San Kim, D.; Dolgy, D.V.; Park, J.W. Sums of finite products of Chebyshev polynomials of the second kind and Fibonacci polynomials. J. Inequal. Appl. 2018, 2018, 148. [CrossRef] [PubMed]

18. Kim, T.; Kim, D.S.; Kwon, J.; Dolgy, D.V. Expressing sums of finite products of Chebyshev polynomials of the second kind and Fibonacci polynomials by several orthogonal polynomials. Mathematics 2018, 6, 210. [CrossRef]

19. Kim, T.; Dolgy, D.V.; Kim, D.S. Representing sums of finite products of Chebyshev polynomials of the second kind and Fibonacci polynomials in terms of Chebyshev polynomials. Adv. Stud. Contemp. Math. (Kyungshang) 2018, 28, 321-335. 
20. Kim, T.; Kim, D.S.; Dolgy, D.V.; Ryoo, C.-S. Representing sums of finite products of Chebyshev polynomials of the third and fourth kinds by Chebyshev polynomials. Symmetry 2018, 10, 258. [CrossRef]

21. Kim, T.; Kim, D.; Victorovich, D.; Ryoo, C. Representing sums of finite products of Legendre and Laguerre polynomials by Chebyshev polynomials. Adv. Stud. Contemp. Math. (Kyungshang) 2018, 28, in press.

22. Doha, E.H.; Abd-Elhameed, W.M.; Alsuyuti, M.M. On using third and fourth kinds Chebyshev polynomials for solving the integrated forms of high odd-order linear boundary value problems. J. Egyptian Math. Soc. 2015, 23, 397-405. [CrossRef]

23. Mason, J.C. Chebyshev polynomials of the second, third and fourth kinds in approximation, indefinite integration, and integral transforms. J. Comput. Appl. Math. 1993, 49, 169-178. [CrossRef]

24. Kim, T.; San Kim, D.; Dolgy, D.V.; Kwon, J. Sums of finite products of Chebyshev polynomials of the third and fourth kinds. Adv. Differ. Equ. 2018, 2018, 283. [CrossRef]

(C) 2018 by the authors. Licensee MDPI, Basel, Switzerland. This article is an open access article distributed under the terms and conditions of the Creative Commons Attribution (CC BY) license (http://creativecommons.org/licenses/by/4.0/). 